\title{
Advance care planning: challenges at the emergency department of a cancer care center
}

\author{
Maria T. Cruz-Carreras ${ }^{1} \cdot$ Patrick Chaftari $^{1} \cdot$ Jayne Viets-Upchurch $^{1}$
}

Received: 24 April 2017 / Accepted: 21 August 2017 / Published online: 16 September 2017

(C) The Author(s) 2017. This article is an open access publication

\begin{abstract}
Introduction Code status discussions form an important part of advance care planning (ACP) as it enables physicians to respect the patient's wishes for end-of-life care. However, in some cases, code status discussions can be challenging causing the physician to go against the patient's wishes and the code of medical ethics. This is especially true in an emergency setting. In this paper, we will discuss three cases of advanced cancer patients, where code status discussions posed challenges to healthcare providers.

Case reports In the first case, the patient was a 26-year-old male diagnosed with advanced osteosarcoma. Code status was discussed with him, while he was still functional, wherein he agreed to a do-not-resuscitate (DNR) order. However, at the time of end-of-life care, despite of previous code status agreement, the patient's mother insisted on full code. As a result, the DNR order was reverted and the patient was intubated. The second case discusses an 83-year-old female patient with metastatic gastric cancer. Code status was extensively discussed with the patient and her son who agreed to sign a DNR order. This case posed a challenge because when the patient's condition deteriorated, her son demanded cardioversion and other aggressive treatment measures without any chest compressions or intubation. In the third case, the patient was a 40-year-old woman with advanced metastatic adenocarcinoma with neuroendocrine features of the parotid. On admission to the ED, as per the patient's wishes expressed by her husband, a DNR/DNI order was placed. However, this order
\end{abstract}

Maria T. Cruz-Carreras

mcruz3@mdanderson.org

1 Department of Emergency Medicine, The University of Texas MD Anderson Cancer Center, Houston, TX, USA had to be reverted when the patient's aunt and sister opposed vehemently to the DNR/DNI order.

Conclusion The three cases demonstrate the challenges that can arise in the implementation of code status order in the ED as it pertains for end-of-life care. In any scenario, respecting the patient's wishes and adherence to the code of medical ethics take precedence over any familial objections arising difficulties with coping.

Keywords Advanced care planning - End of life discussion . Cancer and emergency department

\section{Introduction}

Advance care planning (ACP) refers to a process that permits patients and their family members to discuss with healthcare providers the patient's wishes for end-of-life care [1]. Until recent times, ACP was limited to discussions about the patient's wishes about code status [2]. However, in present healthcare system, ACP is beyond mere code status discussions. It involves detailed discussions regarding several important topics such as discussions about the patient's values, treatment goals, resuscitation and life support, options for palliative care, and future treatment preferences [3]. During ACP, a patient may document his wishes in the form of an advanced directive $(\mathrm{AD})$ and appoint a substitute decision maker on his behalf [3]. ACP provides a means to patient's family members and healthcare providers to ensure that patient's wishes are respected in the event of sudden deterioration in health, which can leave a patient too sick to convey his wishes to others [1, 3]. Despite being a patient-centered initiative, ACP promotes shared decision-making [1].

In general, incorporation of $\mathrm{ACP}$ in healthcare system has shown to increase satisfaction of both, patients and their 
family members [1]. Inclusion of ACP in medical care has also helped healthcare providers in making important decisions that they personally may find quite difficult to undertake [4]. However, incorporation of ACP for the care of grave diseases such as cancer and other advanced conditions can be a sensitive process [3]. Especially, in an emergency setting, discussions about end-of-life care can be difficult and inconsistently interpreted in the future [3]. Hence, inclusion of ACP for end-of-life care for such patients should be based upon evidence of effectiveness.

In this article, we will explore the challenges faced in the advanced care planning of cancer patients in an emergency setting. The difficulties encountered with patients in discussions regarding end-of-life care shall be elucidated through three case presentations.

\section{Case report 1}

The patient was a 26-year-old Hispanic male diagnosed with osteosarcoma. The patient's disease was recurrent and at an advanced stage. He has been found to have metastasis from left tibia, left thigh, and the lungs. The patient had required an above-the-knee amputation as means of palliative resection of his recurrent tumor. The patient's disease was refractory to five lines of chemotherapy and due to lack of insurance coverage issues, his treatment was briefly discontinued.

In 2016, the patient had presented to the Emergency Department (ED) complaining of hemoptysis. The patient was functional at that time, and his investigative tests revealed presence of pulmonary artery involvement by tumor. Following this revelation, code status was discussed with the patient, and he decided to remain full code. After a brief hospital discharge, the patient was re-admitted for complaints of pneumonia and further progression of disease. At this stage, the patient was informed about the dim prognosis and irreversibility of his disease status, and the patient decided to consent to a DNR (do not resuscitate) status including no intubation for mechanical ventilation.

A couple months later, the patient presented to the ED with complaints of shortness of breath and "anxiety attack" as per his mother. The symptoms were present intermittently throughout the day. On examination, the patient was found to be tachypneic, tachycardic, and dyspneic at rest. The chest $\mathrm{X}$-ray revealed progression of disease and right upper lobe (RUL) pneumonia. Care plan was discussed with patient who reported that he wished only for supportive care. Following this, DNR order was signed and the patient was started on supportive care measures. Given his advanced disease and rapid deterioration, a few hours later, the patient lost his decision-making capacity due to hypoxemia and sedation. At that point, patient's mother, as the medical power of attorney, requested to reverse DNR order and demanded intubation with mechanical ventilation support. Despite medical futility and the previous code status agreement with the patient, his mother insisted on full code status for her son. The patient was then intubated and admitted to the intensive care unit (ICU). Finally, 4 days later, the patient's mother agreed to a DNR order and de-escalation of care.

This case was challenging because in spite of the patient's wishes to not be submitted to aggressive measures, his mother protested his decision and insisted the attending physicians on full code status after the patient lost the ability to represent himself. The patient was functional and alert when he had decided on DNR status in case his condition deteriorated. However, the patient's mother went against his wishes and demanded to the physicians to revert the DNR.

This case highlights communication as the essence of providing compassionate care to late stage cancer patients. The patient was functional and alert when he made his DNR choice. He was alert (albeit in distress) upon arrival to the ED when he elected his DNR status and supportive care approach.

A review of the chart reflected only one advance care planning conversation by the oncologist with the patient and his mother. In depth, discussions and documentation at the time the patients speak about their palliative care goals with the oncology teams in clinic are paramount. They allow the ED care team to effectively and smoothly manage stressful situations where a patient is rapidly deteriorating and the family had difficulty in making appropriate decisions because of grieving and denial.

This particular case also underscores the role of physicians as stewards of health care resources. The decision to revert DNR resulted in occupation of an ICU bed during times of high demand for that high level of care. This limited the access to ICU for other patients who were critically ill during the 4 days this patient remained in the ICU intubated.

\section{Case report 2}

The patient was an 83-year-old South Korean woman with locally metastatic gastric cancer and a gastroduodenal ulcerative mass. Along with the primary diagnosis, the patient also had significant comorbidities. These included peripheral vascular disease status post prior stroke and an enlarged abdominal aortic aneurysm. At the time of presentation, the patient was chronically weak, had a poor appetite, and chronically bled from her gastroduodenal tumor. Following admission, the patient was planned to undergo a laparoscopic surgery for the placement of feeding jejunostomy tube and gastrostomy tube. This was followed by chemoradiation therapy for palliation of bleeding and to prevent gastric outlet obstruction.

Besides these interventions, the patient had also been evaluated by the vascular surgical service for her abdominal 
aortic aneurysm. The patient had previously undergone endovascular stenting, but, had a contained endoleak related to her aneurysm and stent procedure. The patient was at relatively high risk for continued leak and rupture. However, due to patient's comorbidities and advanced cancer process, the treatment options were limited, thus, neither open repair nor repeat endovascular repair was considered indicated or feasible at that time.

Code status was discussed extensively with the patient and her son, who was a cardiologist. The patient had clearly expressed her preference in favor of DNR status, including avoid attempts at resuscitation if she went into cardiopulmonary arrest. The patient consented to electrical cardioversion, but she did not consent to chest compressions and prolonged orotracheal intubation if these were to be deemed futile by her treating physician. The patient's son expressed his support for his mother's previously expressed wishes in favor of electrical cardioversion despite DNR status and stated that the two of them had had prior conversations about this issue and her feelings had not changed. Therefore, in accordance with the patient's wishes and in line with the professional recommendation of the attending doctor, a DNR order was signed. Following hospital discharge, the patient had visited the ED multiple times to receive blood transfusions for her chronically bleeding ulcerative malignant mass. In the following months, code status was again discussed with the patient and her family members, wherein, they all remained resolute that their wishes for DNR order had not changed.

This case was challenging because the patient and her physician son demanded cardioversion and other aggressive treatment measures, while, not wanting his mother to have chest compressions or intubation in case of emergency. In practice, aggressive treatment measures such as advanced cardiac life support algorithms are difficult to undertake without intubation and are rarely effective [5]. The goal of electrical cardioversion is to preserve life, restore health, and limit disability. None of those goals could be achieved given her advanced disease status. It was very difficult to convey this to the elderly patient and her grieving son. Despite the fact that the patient's son was a physician, he was too emotionally invested to acknowledge or accept the futility of the requests of his mother and himself to the medical team.

\section{Case report 3}

The patient was a 40-year-old female from Nigeria with advanced metastatic adenocarcinoma with neuroendocrine features of the parotid. The patient presented to the ED in frank respiratory failure with hypercapnea secondary to her advanced pulmonary metastatic disease. On her arrival, the attending physician discussed code status with the patient's husband, who was present by her bedside. The patient's husband expressed that the patient had told him on multiple occasions that she wished to die peacefully and that she did not wish to be submitted to advanced cardiac life support in the event of a cardiopulmonary arrest. If possible, the patient wished to die peacefully at home with her young children. In accordance with the patient's wishes expressed by her husband and legal next of kin, in the ED, a DNR/DNI order was placed in the patient's medical record. However, the patient's aunt and sister, who are both registered nurses in practice, arrived bedside and expressed their disagreement with a DNR/DNI order which had been signed before they arrived to the ED. The patient's aunt and sister were angry, screaming at the patient's husband in their native tongue at the bedside. Meanwhile, the patient rapidly deteriorated hemodynamically and developed respiratory distress. The aunt and sister of the patient became agitated and demanded that the attending physician intubates the patient and requested additional time for the family to visit the patient. Due to the mounting pressure from the rest of the patient's family, the husband gave in to the pressure and asked the medical team to revert the DNR and intubate the patient immediately.

This case was challenging because the patient had expressed DNR wishes when she was alert and oriented. Her husband had tried to respect her wishes and had hence, signed the DNR. But then, as the patient's aunt and sister had stormed into the emergency room and had started to fight with the patient's husband to change the DNR into full code, the husband gave in to the family's pressure and reverted the DNR order into full code status. In this case, the patient's spouse failed in his role as a surrogate decision-maker. A surrogate decision-maker is expected to make decisions using a substituted judgment standard, based on what the patient would want if she had the capacity to express her wishes [6].

\section{Discussion}

As per the American Medical Association (AMA) Principles of Medical Ethics, orders of do-not-attempt-resuscitation (DNAR) direct health care providers to withhold resuscitative measures in accordance with the patient's wishes [7]. DNAR orders are appropriate for any patient who is at risk of cardiopulmonary arrest, regardless of the patient's age or whether the patient is terminally ill [7]. Furthermore, DNAR orders can apply to any care setting, whether in or out of a hospital setting, as long as it is within the constraints of applicable law. As per the AMA code of ethics, physicians have an ethical obligation to implement patient's wishes even if it will result in the patient's death. Moreover, physicians should not permit their personal value judgements to obstruct the implementation of the patient's advance directives [7].

Fair allocation of resources is essential to ensure availability of emergency care to all patients who require it. Physicians 
must strive to gain maximum benefit from available medical resources. If resources are scarce, their distribution should be based on ethically oriented criteria [5].

In the first case, the patient was consulted about code status when he was functional and alert. This was in accordance with AMA code of medical ethics, which advises to ensure the patient's decision-making capacity when initiating discussion about code status. The patient had thus willingly fully agreed to DNR and that was his clear advance directive. However, later, when the patient was re-admitted, his condition had deteriorated considerably and he did not have decision-making capacity. Nonetheless, owing to his advance directive, the physicians knew that the patient's wishes were DNR. However, due to strong insistence from the patient's mother, the advance directive was reversed. This was against the principles of medical ethics and obstructed implementation of the patient's wishes.

In the second case, we have a more complex situation. In this case, the patient's wishes were mostly communicated to the medical teams by her son, a health professional. The patient preferred to have her son speak for her during the medical interviews for cultural reasons and because she trusted and respected his medical expertise. The patient's son was in support of his mother's decision to be DNR but he was adamant that his mother should receive some selective aggressive treatments such as electrical cardioversion, and the patient accepted her son's position on this matter. Initiation of aggressive therapies without any intubation or CPR posed the issue of futility and waste of resources to the treating physicians.

In the third case, the patient's wishes were clearly expressed by her husband, which was to not be submitted to aggressive, futile measures. However, the belligerent behavior of the patient's aunt and sister and their continued insistence to have the sick cancer patient remains full code, forced the patient's husband to reverse the advance directive he had received from his wife. This was also against the code of medical ethics, as the patient's wishes, as expressed by her next of kin, were not respected.

The three case scenarios, present to us the difficulty of code status discussions for cancer patients in an emergency setting. Discussions about ACP, including code status, should not be conducted in haste. As per the AMA code of medical ethics, ACP is a comprehensive process and physicians should routinely engage their patients to consider their values, ethics, and future treatment preferences in making decisions about endof-life care. However, in an emergency setting, physicians do not have the opportunity or the time to conduct detailed discussions with the patient or their family members regarding the pros and cons of medical resuscitative measures in case the patient's health deteriorates. Especially in cancer care, most patients arriving at the ED are critically ill and it must be decided at the spur of the moment, whether the patients will be DNR or full code. Furthermore, even if, the patient's wishes are clearly known to the physician in the form of an advance directive as in Case 1 or if the patient's wishes are clearly expressed by their next of kin as in 3, the attending physicians do not have time to consult other members of the patient's family and discuss code status decisions with them. Due to this drawback, as seen in Case 1 and 3, though, the patients had clear directives; the physicians had to go against their wishes owing to continued pressure from their family members who opposed the patient's directives. This is against the code of medical ethics as in any scenario the physician must not shift from implementing the patient's wishes. However, resuscitative measure is a sentimental matter and physicians under the firm pressure and insistent demands of a patient's family members can be forced to go against the patient's wishes after the patient loses the capacity to represent himself or herself.

\section{Compliance with ethical standards}

Conflict of interest The authors declare that they have no conflict of interest.

Open Access This article is distributed under the terms of the Creative Commons Attribution-NonCommercial 4.0 International License (http:// creativecommons.org/licenses/by-nc/4.0/), which permits any noncommercial use, distribution, and reproduction in any medium, provided you give appropriate credit to the original author(s) and the source, provide a link to the Creative Commons license, and indicate if changes were made.

\section{References}

1. Luckett T, Sellars M, Tieman J et al (2014) Advance care planning for adults with CKD: a systematic integrative review. Am J Kidney Dis 63(5):761-770

2. Houben CHM, Martijn AS, Groenen MTJ, Wouters EFM, Janssen DJA (2014) Efficacy of advance care planning: a systematic review and meta-analysis. JAMDA 15:477-489

3. Jones L, Harrington J, Barlow C et al (2011) Advance care planning in advanced cancer: can it be achieved? An exploratory randomized patient preference trial of a care planning discussion. Palliat Support Care 9:3-13

4. Calam B, Andrew R (2000) CPR or DNR? End-of-life decision making on a family practice teaching ward. Can Fam Physician 46: 340-346

5. Guidelines 2000 for cardiopulmonary resuscitation and emergency cardiovascular care. Part 2: ethical aspects of CPR and ECC. Circulation 102(8 Suppl):I12-I21

6. Braddock CH, Clark JD (2014) Do Not Resuscitate (DNAR) Orders. Ethics in Medicine-Bioethics Topics 2014. Available from: https:// depts.washington.edu/bioethx/topics/dnr.html

7. American Medical Association (2016) AMA Code of medical ethicsCh 5: Opinions on caring for patients at the end of life. https://www. ama-assn.org/delivering-care/ama-code-medical-ethics 